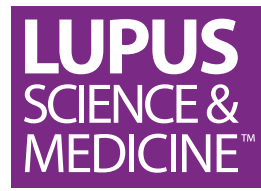

To cite: Fortner KA, Blanco LP, Buskiewicz I, et al. Targeting with MitoQ reduces NET formation and kidney disease in lupus-prone MRL-Ipr mice. Lupus Science \& Medicine 2020;7:e000387. doi:10.1136/ lupus-2020-000387

KAF and LPB contributed equally.

Received 22 January 2020 Revised 6 March 2020 Accepted 13 March 2020

\title{
Targeting mitochondrial oxidative stress with MitoQ reduces NET formation and kidney disease in lupus-prone MRL- lpr mice
}

\author{
Karen A Fortner, ${ }^{1}$ Luz P Blanco (D) , ${ }^{2}$ Iwona Buskiewicz, ${ }^{3}$ Nick Huang, ${ }^{4}$ \\ Pamela C Gibson, ${ }^{5}$ Deborah L Cook, ${ }^{5}$ Hege L Pedersen, ${ }^{2}$ Peter S T Yuen, ${ }^{6}$ \\ Michael P Murphy (D) , Andras Perl, ${ }^{4}$ Mariana J Kaplan (D) , Ralph C Budd (D) ${ }^{1}$
} mitochondrial oxidative stress

\begin{abstract}
Objectives Recent investigations in humans and mouse models with lupus have revealed evidence of mitochondrial dysfunction and production of mitochondrial reactive oxygen species (mROS) in T cells and neutrophils. This can provoke numerous cellular changes including oxidation of nucleic acids, proteins, lipids and even induction of cell death. We have previously observed that in T cells from patients with lupus, the increased mROS is capable of provoking oligomerisation of mitochondrial antiviral stimulator (MAVS) and production of type I interferon (IFN-I). mROS in SLE neutrophils also promotes the formation of neutrophil extracellular traps (NETs), which are increased in lupus and implicated in renal damage. As a result, in addition to traditional immunosuppression, more comprehensive treatments for lupus may also include non-immune therapy, such as antioxidants.

Methods Lupus-prone MRL-Ipr mice were treated from weaning for 11 weeks with the mitochondria-targeted antioxidant, MitoQ $(200 \mu \mathrm{M})$ in drinking water. Mice were then assessed for ROS production in neutrophils, NET formation, MAVS oligomerisation, serum IFN-I, autoantibody production and renal function.

Results MitoQ-treated mice manifested reduced neutrophil ROS and NET formation, decreased MAVS oligomerisation and serum IFN-I, and reduced immune complex formation in kidneys, despite no change in serum autoantibody .

Conclusions These findings reveal the potential utility of targeting mROS in addition to traditional immunosuppressive therapy for lupus.
\end{abstract}

\section{INTRODUCTION}

SLE is a complex and heterogeneous autoim(C) Author(s) (or their employer(s)) 2020. Re-use permitted under CC BY. Published by BMJ.

For numbered affiliations see end of article.

Correspondence to

Dr Ralph C Budd; ralph.budd@ med.uvm.edu mune syndrome characterised by numerous abnormalities, including various autoantibodies, the appearance of unusual CD4-CD8TCR- $\alpha \beta^{+}$cells, altered metabolism of lymphocytes, a type I interferon (IFN-I) gene signature in peripheral blood mononuclear cells (PBMC), increased formation of neutrophil extracellular traps (NETs), and deposition of immunoglobulins and complement at renal glomeruli. ${ }^{1-10}$ Each of these immune abnormalities may provoke different aspects of the disease, and each also may be driven by different environmental and genetic aberrations. Recent work has also revealed the importance of non-immune factors, such as oxidative stress, in the development of endorgan damage in SLE, shifting the paradigm of SLE pathogenesis from that of a disease provoked solely by a disturbed immune system. ${ }^{17}$ Thus, fully effectively treatment of SLE may require inhibiting pathways in addition to those targeted by traditional immunosuppressive medications.

Neutrophil activation in response to glomerular immune complexes contributes to lupus glomerulonephritis, in part through the production of mitochondrial reactive oxygen species (mROS) that can directly injure tissue through oxidation of lipids, proteins, DNA and induction of apoptosis. ${ }^{112}$ We have shown previously that immune complex activation of neutrophils can also lead to the formation of NETs, which can release oxidised genomic and mitochondrial DNA and promote the production of IFN-I. ${ }^{1012}$ Furthermore, a subset of low-density granulocytes from patients with SLE has an enhanced capacity to form NETs in an mROS-dependent manner, and these structures have been observed in the kidneys of patients with lupus nephritis as well as in other lupus-affected tissues. ${ }^{1113}$

We have also previously observed that $\mathrm{T}$ lymphocytes from patients with SLE manifest mitochondrial dysfunction as evidenced by enlarged mitochondria and elevated mROS that can induce oxidative damage. ${ }^{14}$ The mROS triggers the spontaneous oligomerisation of the mitochondrial antiviral stimulator (MAVS) protein and downstream IFN-I 
production. ${ }^{15}$ This was likely due in part to induction of cysteine disulfide bonding in the caspase recritment domain (CARD) of MAVS, since a C79F polymorphism of MAVS known to be associated with milder SLE ${ }^{16}$ did not efficiently oligomerise nor induce IFN-I with mROS. ${ }^{15}$ In addition, we observed that oligomerised MAVS is reversed in vitro in the presence of the mitochondrial antioxidant, MitoQ. ${ }^{15}$ Consequently, mROS and resulting oxidative damage emerge as possible driving forces in certain aspects of SLE, and hence represent a target for therapy.

The process that promotes the mitochondrial abnormalities in human SLE T cells is unknown. Hence, we examined whether a similar phenomenon of enlarged mitochondria and spontaneous MAVS oligomerisation might occur in any of the T-cell subsets of lupus-prone MRL-lpr mice. T cells in these mice accumulate in large numbers in lymphoid organs through dysregulated homeostatic proliferation that is enhanced in the absence of the death receptor, Fas (CD95) ${ }^{17}$ The dysregulation of $\mathrm{T}$ cells includes the emergence with age of an increasingly large proportion of polyclonal $\mathrm{CD} 4^{-} \mathrm{CD} 8^{-} \mathrm{TCR}-\alpha \beta^{+}$cells that derive from $\mathrm{CD} 8^{+}$precursors during homeostatic proliferation. ${ }^{17}$ A CD $4^{-} \mathrm{CD} 8^{-}$TCR- $\alpha \beta^{+}$subset also occurs in human SLE. ${ }^{34}$ Similar to human SLE T cells, the $l p r$ $\mathrm{CD} 4^{-} \mathrm{CD} 8^{-} \mathrm{TCR}-\alpha \beta^{+}$subset also manifested enlarged mitochondria and spontaneous MAVS oligomerisation. We thus investigated further the ability of the mitochondriatargeted antioxidant Mito $Q$ in vivo to reverse mROS and NET formation, MAVS oligomerisation, as well as to test its therapeutic potential on lupus disease manifestations in MRL-lpr mice.

\section{METHODS \\ Mice}

Mice were bred and housed in the Association for Assessment and Accreditation of Laboratory Animal Care International-approved animal facilities of The University of Vermont Larner College of Medicine. Original breeding pairs of MRL/MpJ-Fas ${ }^{l p r}$ (MRL-lpr) mice were obtained from Jackson Laboratory (Bar Harbor, Maine, USA). All breeding and animal studies were conducted in accordance with the policies of The University of Vermont's Animal Care and Use Committee.

\section{MitoQ treatment}

Mice were weaned at 4 weeks and placed on either drinking water alone or $200 \mu \mathrm{M}$ MitoQ (MitoQ Auckland, NZ) in drinking water. Bottles were changed weekly. After 11 weeks of treatment, mice were euthanised and kidneys harvested for histological analysis. Brachial and axillary lymph nodes were assessed for cell number and lymphocyte subsets were determined by flow cytometry. Neutrophils were purified from bone marrow as previously described. ${ }^{10}$ Serum was obtained for autoantibodies, creatinine and blood urea nitrogen (BUN). Urine was obtained for creatinine measurement.

\section{Lymphocyte preparation}

Single cell suspensions of lymph nodes were prepared in Roswell Park Memorial Institute (RPMI) 1640 (CellGro, Corning, Manassas, Virginia, USA) containing 25 mM 4-(2hydroxyehtyl)-1-piperazineethanesulfonic acid (HEPES), $5 \% \mathrm{v} / \mathrm{v}$ bovine calf serum (HyClone, Logan, Utah, USA), $5 \times 10^{-5}$ M $\beta$-mercaptoethanol (Sigma, St. Louis, Missouri, USA), $100 \mathrm{U} / \mathrm{mL}$ penicillin and $100 \mathrm{U} / \mathrm{mL}$ streptomycin (Life Technologies-Invitrogen; Grand Island, New York, USA) (RPMI $/ 5 \%$ bovine calf serum (BCS)). $\mathrm{CD}^{+}$and $\mathrm{CD} 4^{-} \mathrm{CD} 8^{-} \mathrm{TCR} \alpha \beta^{+} \mathrm{T}$ cells were isolated by negative selection. Lymph node cells were incubated with the appropriate antibodies (see below) for $30 \mathrm{~min}$ on ice. After washing, the cells were incubated by rocking with goat anti-rat and goat anti-mouse IgG-coated beads (Qiagen, Valencia, California, USA) for $45 \mathrm{~min}$ at $4^{\circ} \mathrm{C}$. Antibodycoated cells were removed by magnetic depletion. To obtain $\mathrm{CD}^{+} \mathrm{T}$ cells, cell suspensions were incubated with anti-class II (3F12), anti-CD11b (M1/70), anti-NK1.1 (PK136), anti-kappa (187.1), anti-CD4 (GK1.5) and antiCD45R (B220, RA3GB2). To isolate CD $4^{-} \mathrm{CD} 8^{-} \mathrm{TCR} \alpha \beta^{+} \mathrm{T}$ cells, cells were incubated with anti-class II (3F12), antiCD11b (M1/70), anti-NK1.1 (PK136), anti-kappa (187.1), anti-CD4 (GK1.5) and anti-CD8 (Tib105).

\section{Antibodies and flow cytometry}

The following antibodies to murine cell surface proteins were purchased from BD Biosciences (San Jose, California, USA): allophycocyanin (APC)-conjugated antiTCR $\beta$ and Pacific Blue-conjugated anti-CD45R (B220). The following antibodies were purchased from Life Technologies-Invitrogen: phycoerythrin (PE)-conjugated anti-CD44, PE-Texas Red-conjugated anti-CD4, PE Cy5.5conjugated anti-CD8 and Pacific Orange-conjugated anti-CD45. The following antibodies were purchased from BioLegend (San Diego, California, USA): Alexa 647-conjugated anti-TCR $\gamma \delta$, Alexa 700-conjugated antiCD19 and Pacific Blue-conjugated anti-CD19. Live Dead Fixable Blue was purchased from Life TechnologiesMolecular Probes. Lyophilised rat IgG and hamster IgG (MP Biochemicals, Solon, Ohio, USA) were resuspended in phosphate-buffered saline (PBS) and stored at $-80^{\circ} \mathrm{C}$.

For direct staining, single cell suspensions were washed with cold $\left(4^{\circ} \mathrm{C}\right) \mathrm{PBS}$ and then incubated with Live Dead Fixable Blue Stain (Life Technologies-Molecular Probes, Eugene, Oregon, USA) in PBS for $30 \mathrm{~min}$ at $4^{\circ} \mathrm{C}$. The cells were washed with cold PBS containing $1 \% \mathrm{w} / \mathrm{v}$ bovine serum albumin (BSA) fraction $\mathrm{V}$ (Sigma) (PBS/1\% BSA) and then incubated with a mixture of rat IgG and hamster $\operatorname{IgG}(50 \mu \mathrm{g} / \mathrm{mL}$ each $)$ for $30 \mathrm{~min}$ at $4^{\circ} \mathrm{C}$. After washing, the cells were incubated with the appropriate antibodies in PBS/1\% BSA, washed and fixed with freshly made $1 \% \mathrm{v} / \mathrm{v}$ methanol-free formaldehyde (Ted Pella, Redding, California, USA) in PBS/1\% BSA. Flow cytometry was performed on an LSR II (BD Biosciences) and the data were analysed using FloJo software (Tree Star, Ashland, Oregon, USA). 
Analysis of mitochondrial morphology by transmission electron microscopy

Cells were fixed for 1 hour at $65^{\circ} \mathrm{C}$ in $2 \%$ paraformaldehyde and $2.5 \%$ glutaraldehyde (Polysciences, Warrington, Pennsylvania, USA) in $100 \mathrm{mM}$ sodium cacodylate buffer ( $\mathrm{pH}$ 7.2). Samples were washed in cacodylate buffer and then postfixed for 1 hour in $1 \%$ osmium tetroxide (Polysciences). Samples were then extensively rinsed in distilled $\mathrm{H}_{2} \mathrm{O}$ before undergoing en bloc staining for 1 hour with $1 \%$ aqueous uranyl acetate (Ted Pella). After several rinses in distilled $\mathrm{H}_{2} \mathrm{O}$, the samples were dehydrated in a graded series of ethanol and then embedded in Eponate 12 resin (Ted Pella). Sections (95 nm in thickness) were cut with an Ultracut UC7 ultramicrotome (Leica Microsystems, Wetzlar, Germany), stained with uranyl acetate and lead citrate, and viewed on a JEOL 1400 transmission electron microscope (JEOL USA) equipped with an XR611 high-resolution, 11-megapixel mid-mount charge-coupled device camera (Advanced Microscopy Techniques, Woburn, Massachusetts, USA).

\section{Semidenaturing detergent agarose gel electrophoresis (SDD-} AGE) for the detection of MAVS oligomers

SDD-AGE was performed according to a published protocol with minor modifications. ${ }^{18}$ Briefly, mitochondria were resuspended in sample buffer $(0.5 \times$ tris-borate EDTA (TBE), 10\% glycerol, 2\% sodium dodecyl sulfate (SDS) and $0.0025 \%$ bromophenol blue) and loaded onto a vertical $1.5 \%$ agarose gel. After electrophoresis in running buffer ( $1 \times \mathrm{TBE}, 0.1 \% \mathrm{SDS})$ for $35 \mathrm{~min}$ with

A

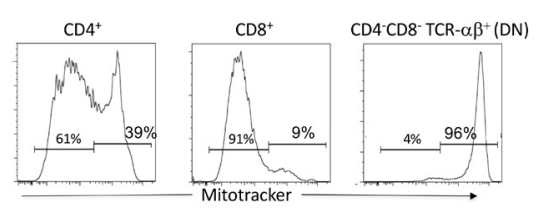

B
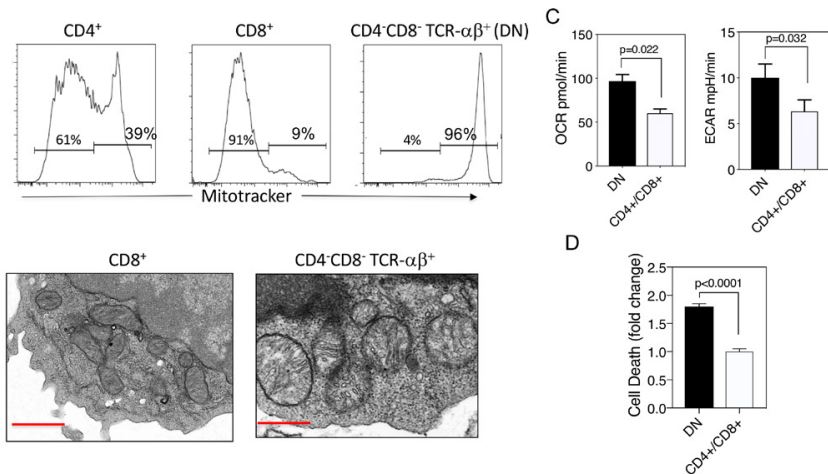

Figure 1 MRL-Ipr CD4 ${ }^{-} \mathrm{CD} 8^{-}$TCR- $\alpha \beta^{+}$cells have enlarged mitochondria and increased oxidative and glycolytic metabolism. (A) Lymph node cells from MRL-lpr mice were analysed by flow cytometry for the expression of TCR- $\alpha \beta^{+}$, CD4, CD8 and mitochondrial mass using MitoTracker. (B) Electron micrographs (12000x) of mitochondria from MRL-Ipr $\mathrm{CD} 8^{+}$or $\mathrm{CD}^{-} \mathrm{CD}^{-} \mathrm{T}$ cells. Red bar inset represents $500 \mathrm{~nm}$. (C) OCR and ECAR for freshly isolated MRL-Ipr $\mathrm{CD} 4^{+}$plus $\mathrm{CD}^{+} \mathrm{T}$ cells or $\mathrm{CD} 4^{-} \mathrm{CD} 8^{-} \mathrm{T}$ cells determined in extracellular flux analyser. Shown are means \pm SEM of five replicates of each subset. (D) Spontaneous cell death following a 2hour incubation in complete medium at $37^{\circ} \mathrm{C}$. Findings and means \pm SEM for three experiments normalised to death rate of $\mathrm{CD}^{+}$plus $\mathrm{CD}^{+}$subset. Results are representative of three experiments. Statistical analysis was by unpaired t-test. DN, double negative; ECAR, extracellular acidification rate; OCR, oxygen consumption rate. a constant voltage of $75 \mathrm{~V}$ at $4^{\circ} \mathrm{C}$, proteins were transferred to polyvinylidene difluoride (PVDF) membranes with a Trans-Blot Turbo Transfer System in preparation for western blot analysis. PVDF membranes were blocked in Tris-buffered saline and 5\% non-fat powdered milk and analysed with MAVS-specific antibody (Santa Cruz Biotechnology, Dallas, Texas, USA). Immunoreactive proteins were visualised with horseradish peroxidaselabelled conjugates (Jackson ImmunoResearch, West Grove, Pennsylvania, USA) and developed with Clarity Western ECL Substrate (Bio-Rad, Hercules, California, USA). Chemiluminescence was detected and recorded with a Bio-Rad Chemidoc instrument. Densitometric measurements were performed in Image Lab image acquisition and analysis software (Bio-Rad).

\section{Metabolism analysis}

Real-time analysis of extracellular acidification rates and oxygen consumption rates were measured with the XFe96 extracellular flux analyser (Agilent Technologies, Santa Clara, California, USA) according to the manufacturer's specifications. Metabolic profiles were measured under basal conditions in non-buffered Dulbecco's Modified Eagle Medium (Sigma) containing $25 \mathrm{mM}$ glucose, $2 \mathrm{mM}$ L-glutamine and $1 \mathrm{mM}$ sodium pyruvate, in response to $1 \mathrm{mM}$ oligomycin, $0.5 \mathrm{mM}$ trifluoromethoxy carbonylcyanide phenylhydrazone (FCCP) and $1 \mathrm{mM}$ rotenone $/ 1 \mathrm{mM}$ antimycin. Analysis was performed with the Wave Software V.2.4 or V.2.6 (Agilent Technologies).

\section{Quantification of NETs and mROS in bone marrow neutrophils}

The isolation of bone marrow-derived neutrophils and quantification of NETs and mROS were performed as previously described. ${ }^{10}$ Briefly, hindlimb marrow neutrophils were purified by Percoll gradient. Cells were seeded in a 96-well plate $(200000$ cells $/ 100 \mu \mathrm{L} /$ well $)$ in triplicates for each dye and allowed to form NETs in the presence of SYTOX (externalised DNA, $1 \mu \mathrm{M}$ final concentration), Quant-It Picogreen (total DNA stock solution diluted 1:250) and MitoSox (200 ng/mL) (all from Thermo Fisher, Waltham, Massachusetts, USA). Fluorescence was measured at different time points for each dye, at the earliest time point $485 / 520$ (Picogreen), 1 hour $510 / 580$ (MitoSox) and 2 hour 486/520 (SYTOX), using a FLUOstar Omega BMG Labtech (Cary, North Caroline, USA) plate reader. Picogreen measurement was used as the initial number of cells or total DNA.

\section{Autoantibody quantification}

Serum concentrations of autoantibodies were determined using commercially available ELISA kits (Alpha Diagnostic International, Texas, USA). Serum was diluted (1:125) in non-specific binding (NSB) buffer and the assay done following manufacturer's instructions.

Assessment of kidney histology and function

Renal immune complex deposition was quantified as previously described $^{10}$ using an Alexa Fluor 594F(ab') 2goat antimouse IgG (Thermo Fisher) and fluorescein 
A

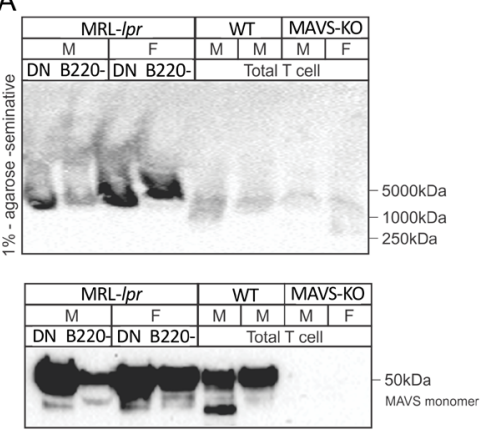

B

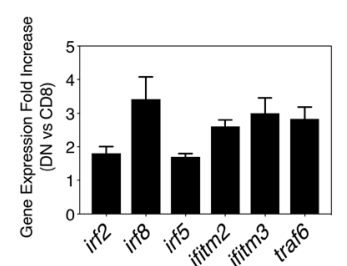

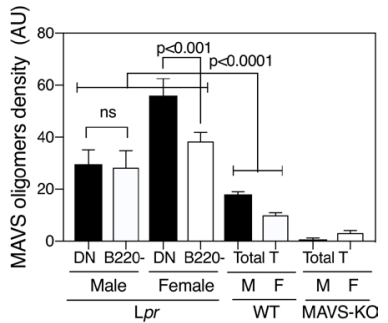

C

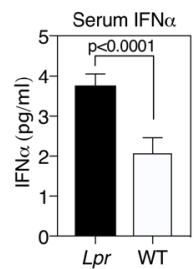

Figure 2 MRL-Ipr CD4 $4^{-} \mathrm{CD} 8^{-} \mathrm{TCR}-\alpha \beta^{+}$cells manifest spontaneously oligomerised mitochondrial antiviral stimulator (MAVS) and increased expression of type I interferon (IFNI)-stimulated genes (ISG) and serum IFN-I. (A) Lymph node cells from MRL-Ipr mice were separated into $\mathrm{CD}^{-} \mathrm{CD} 8^{-} \mathrm{TCR}-$ $\alpha \beta^{+}$(double-negative (DN)) and $\mathrm{CD} 4^{+}$plus $\mathrm{CD}^{+}\left(\mathrm{B} 220^{-}\right)$ subsets and cell lysates prepared. Comparisons were made with lymph node cells from $\mathrm{MRL}^{+/+}$wild-type (WT) or MAVSknockout (KO) mice. Lysates were subjected to $1 \%$ agarose gels (upper panel) to selectively visualise MAVS oligomers, or SDS-PAGE (sodium dodecyl sulfate-polyacrylamide gel electrophoresis; lower panel) to visualise total MAVS monomer levels. (B) Fold increase in RNA expression for several ISG comparing female MRL-Ipr DN to naive $\mathrm{CD} 8^{+} \mathrm{CD} 44^{\text {low }}$ cells of the same mice. RNA was hybridised to Affymetrix GeneChip Mouse 430 2.0. Results are from five untreated 10-week-old mice per experiment and performed three times. (C) Serum levels of IFN-I in female $\mathrm{MRL}^{+/+}$WT and MRL-Ipr mice. Shown are the mean \pm SEM of five mice of each type. Statistical analysis was by unpaired t-test. Results were very similar in three experiments. F, female; $M$, male.

isothiocyanate (FITC)-antimurine C3 antibody (Immunology Consultants Laboratories, Portland, Oregon USA). Nuclei were stained with Hoechst (1:500; Life Technologies, Carlsbad, California, USA). For quantification, three random images were obtained from each stained frozen section. The images were analysed with Image J software selecting the glomerular compartment to quantify mean pixels for each fluorescence channel used.

To quantify serum creatinine and eliminate the influence of chromogens in mouse serum that interfere with the classic Jaffe method for creatinine detection, an high performance liquid chromatography assay was used as previously described. ${ }^{19}$ Briefly, $5 \mu \mathrm{L}$ serum were treated with $0.5 \mathrm{~mL}$ acetonitrile, centrifuged at $4^{\circ} \mathrm{C}$ at $13000 \times g$ for $20 \mathrm{~min}$, and supernatants were dried by SpeedVac and resuspended in mobile phase $(5 \mathrm{mM}$ sodium acetate, $\mathrm{pH}$ 5.1). Duplicates were run on a $100 \times 4.1 \mathrm{~mm}$ PRP-X200 column (Hamilton, Reno, Nevada, USA) and isocratically eluted at $2 \mathrm{~mL} / \mathrm{min}$ in an Agilent 1100 System, with

ultraviolet detection at $234 \mathrm{~nm}$. Absolute quantitation was determined with a standard curve of $2-50 \mathrm{ng}$ creatinine $\left(r^{2}=0.999\right)$.

\section{Statistical analysis}

Statistical analyses were performed using the graphing software Prism V.7 (GraphPad Software, La Jolla, California, USA). The following statistical tests were used: paired and unpaired t-test when comparing two conditions, one-way analysis of variance (ANOVA) with Tukey's test for correction for multiple comparisons when comparing multiple conditions and two-way ANOVA with Sidak test for correction for multiple comparisons when comparing multiple variables across multiple conditions. All data met the assumptions of the statistical tests used and variation among the compared groups was similar.

\section{RESULTS}

MRL-Ipr CD4-CD8 ${ }^{-}$TCR- $\alpha \beta^{+}$cells have enlarged mitochondria, increased oxygen consumption and glycolysis

Our previous observations in human SLE T cells revealed that they manifest enlarged mitochondria, mROS production and spontaneous MAVS oligomerisation. ${ }^{51415}$ We thus examined lupus-prone MRL-lpr mouse T cells for similar features. Initial analysis revealed that $\operatorname{lpr} \mathrm{CD}^{+}$ $\mathrm{T}$ cells, the precursors of the $\mathrm{CD} 4^{-} \mathrm{CD} 8^{-} \mathrm{TCR}_{-} \alpha \beta^{+} \mathrm{T}$ cells, ${ }^{172021}$ contained relatively low mitochondrial mass,
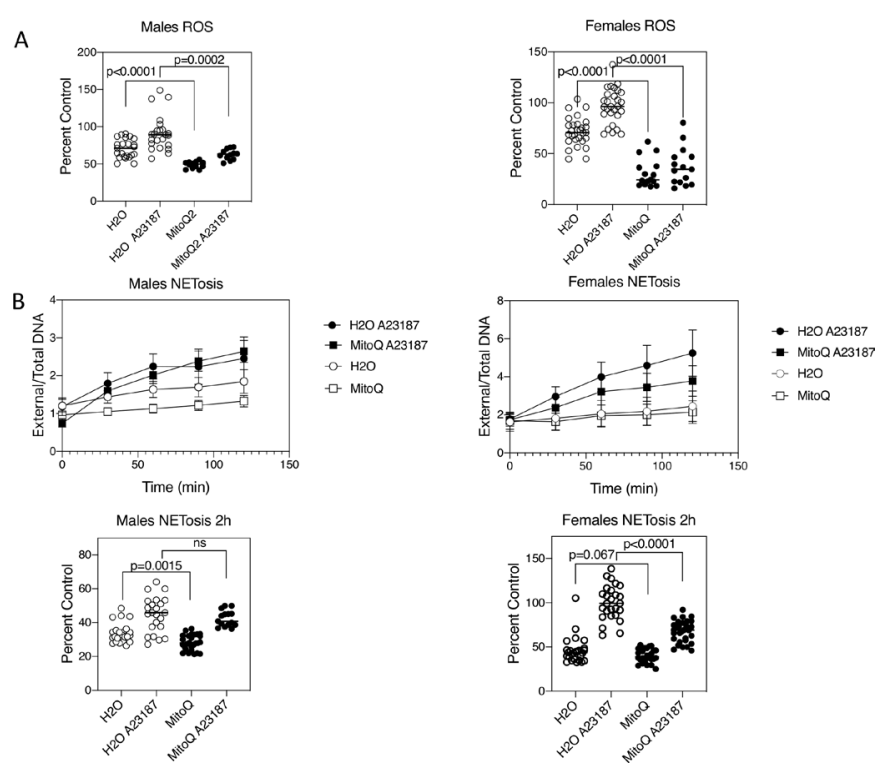

Figure 3 In vivo MitoQ treatment reduces levels of reactive oxygen species (ROS) and neutrophil extracellular trap (NET) formation in neutrophils. Mice (eight mice per treatment group) were administered either drinking water alone, or water containing MitoQ $(200 \mu \mathrm{M})$ for 11 weeks following weaning. Neutrophils were purified from bone marrow and assayed for (A) ROS production and (B) NET formation either spontaneously or following activation with A23187. Per cent control refers to the comparison of each value to the mean of female neutrophils with A23187 activation. Shown are points for individual mice and their mean for three experiments. Statistical analysis was unpaired t-test. ns, non-significant. 
A
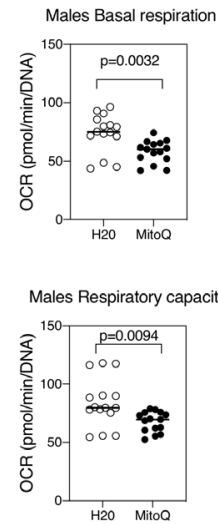
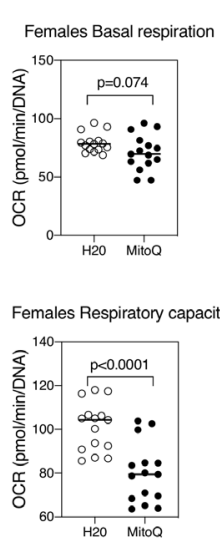

B
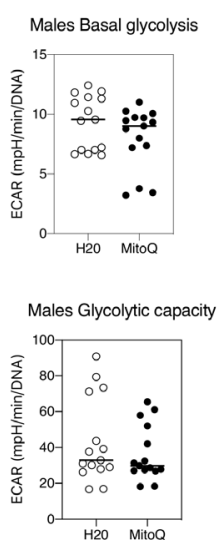

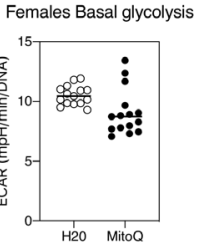

Females Glycolytic capacity

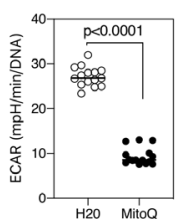

Figure 4 Reduced oxygen consumption and glycolysis following in vivo MitoQ treatment. Extracellular flux analysis for (A) oxygen consumption rate $(\mathrm{OCR})$ and $(\mathrm{B})$ glycolysis as measured by extracellular acidification rate (ECAR) for neutrophils from mice (six mice per group, two experiments) administered water alone or MitoQ $(200 \mu \mathrm{M})$ in drinking water. Shown are mean and statistical analysis by unpaired t-test. Results are representative of three experiments.

using MitoTracker and flow cytometry, whereas the CD4 ${ }^{-}$ CD8 ${ }^{-}$TCR- $\alpha \beta^{+} \mathrm{T}$ cells had markedly higher mitochondrial mass relative to the $\mathrm{CD} 8^{+} \mathrm{T}$ cells (figure 1A). Further analysis by electron microscopy revealed that, similar to human SLE T cells ${ }^{514}$ the $l p r$ CD $4^{-} \mathrm{CD} 8^{-}$TCR- $\alpha \beta^{+} \mathrm{T}$ cells contained very large and rounded mitochondria, in contrast to the more typical elongated mitochondria of the $\mathrm{CD}^{+}$subset (figure 1B). This paralleled greater rates of oxygen consumption and glycolysis in the $\mathrm{CD} 4^{-} \mathrm{CD} 8^{-}$ TCR- $\alpha \beta^{+}$subset, as detected by Seahorse extracellular flux analysis (figure 1C). The increased aerobic glycolysis of $\mathrm{CD} 4^{-} \mathrm{CD} 8^{-} \mathrm{TCR}-\alpha \beta^{+} \mathrm{T}$ cells is consistent with the known rapid proliferation by this subset in vivo. ${ }^{22}$ This is paralleled by increased spontaneous cell death of the $\mathrm{CD} 4^{-} \mathrm{CD} 8^{-}$TCR- $\alpha \beta^{+} \mathrm{T}$ cells compared with the $\mathrm{CD} 4^{+}$and $\mathrm{CD}^{+} \mathrm{T}$ cell subsets (figure $1 \mathrm{D}$ ), consistent with previous
A
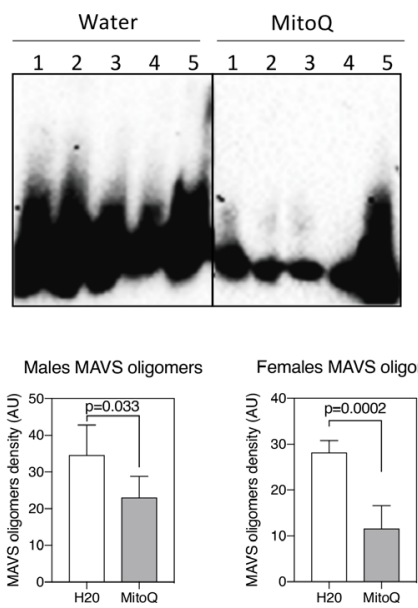

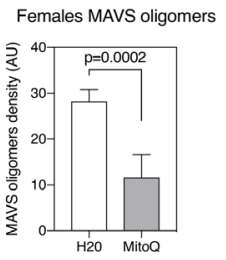

B
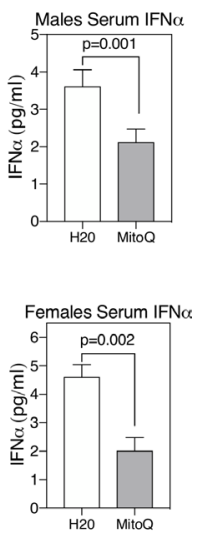

Figure 5 Reduced mitochondrial antiviral stimulator (MAVS) oligomerisation and serum interferon (IFN)- $\beta$ with MitoQ. (A) MAVS oligomers were assessed by western blot from non-denaturing agarose gels as described in the Materials and methods section. Shown are results from five mice per treatment group. Graphs indicate density of MAVS oligomers. (B) Serum IFN $\alpha$ as measured by ELISA. Shown are mean \pm SEM and statistical analysis by unpaired t-test. Results are representative of two experiments. observations that high levels of glycolysis in $\mathrm{T}$ cells, including $\mathrm{CD} 4^{-} \mathrm{CD} 8^{-}$TCR- $\alpha \beta^{+}$T cells, drives high levels of active caspase-3, rendering them prone to cell death. ${ }^{23} 24$ Such increased cell death could contribute to the inflammatory response in these mice.

Given these parallels between T cells from human SLE and the $\mathrm{CD} 4^{-} \mathrm{CD} 8^{-}$TCR- $\alpha \beta^{+} \mathrm{T}$ cells of MRL-lpr mice, we examined whether they also manifested evidence of spontaneous MAVS oligomerisation and activation of IFN-I genes. Indeed, the $\mathrm{CD} 4^{-} \mathrm{CD} 8^{-} \mathrm{TCR}-\alpha \beta^{+}\left(\mathrm{B} 220^{+}\right)$subset contained MAVS oligomers, which were diminished in the $\mathrm{CD} 4^{+}$and $\mathrm{CD}^{+}\left(\mathrm{B} 220^{-}\right)$fraction and non-existent in wild-type mice (figure 2A). This paralleled the upregulation of several IFN-I-stimulated genes in the $\mathrm{CD} 4^{-} \mathrm{CD} 8^{-}$ TCR- $\alpha \beta^{+} \mathrm{T}$ cells compared with the $\mathrm{CD} 8^{+}$precursors (figure 2B). Consistent with these findings, MRL-lpr mice had higher levels of serum IFN $\alpha$ compared with wild-type mice (figure 2C). Collectively, these findings suggest that mROS may drive MAVS oligomerisation in $\operatorname{lpr} \mathrm{CD} 4^{-} \mathrm{CD}^{-}$ TCR- $\alpha \beta^{+}$T cells as it does in human SLE. We thus considered that mROS and possible oxidative damage might also drive some of the disease manifestations in MRL$l p r$ mice, and elected to treat the mice in vivo with the mitochondria-targeted antioxidant MitoQ.

\section{In vivo MitoQ treatment of MRL-Ipr mice reduces ROS} production and NET formation by neutrophils

Increased production of mROS and NET formation has been observed in neutrophils of patients with SLE. ${ }^{10}{ }^{11}$ As NET formation in human lupus is, at least in part, driven by mROS production, we initially examined whether the mitochondria-targeted antioxidant, Mito $Q$, would reduce neutrophil mROS and NET formation. MitoQ contains an antioxidant, ubiquinonol, coupled to a triphenylphosphonium moiety containing three phenyl groups to promote membrane permeabilisation, combined with a central positively charged phosphorous that draws the compound toward negative charges. As mitochondria are about $150-170 \mathrm{mV}$ negative compared with the cytosol, which is itself a further $30-60 \mathrm{mV}$ negative compared 

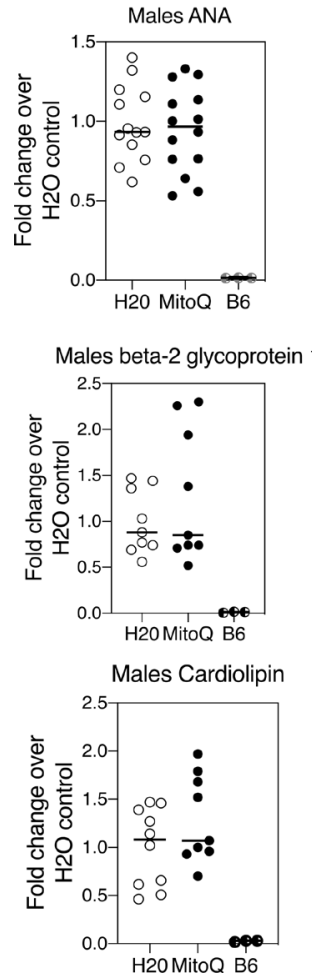

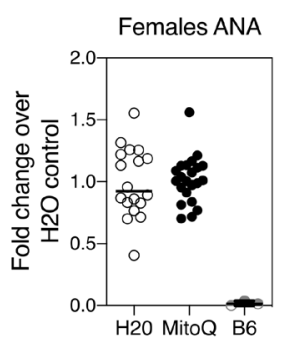

Females beta-2 glycoprotein 1
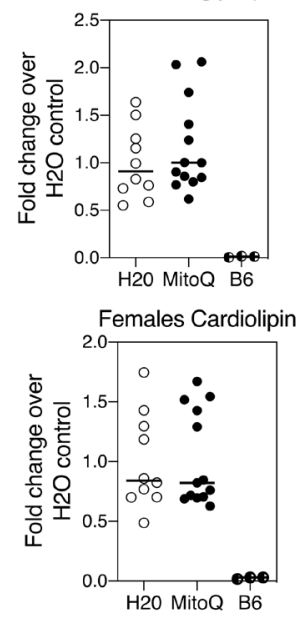

Figure 6 MitoQ does not alter autoantibody titres in MRL-Ipr mice. MRL-Ipr mice received water only or water containing MitoQ for 11 weeks. Serum autoantibody titres were measured by ELISA. Results shown are the combined findings of three experiments normalised to the water-only controls. Numbers of MRL-lpr mice in each treatment group $\left(n=\mathrm{H}_{2} \mathrm{O} /\right.$ MitoQ) were: males ANA $(n=13 / 14)$, males beta-2 glycoprotein $1(n=9 / 9)$, males cardiolipin $(n=10 / 9)$, females ANA $(n=18 / 22)$, females beta-2 glycoprotein $1(n=10 / 13)$, and females cardiolipin $(n=10 / 13)$.

with the extracellular environment, Mito compounds are concentrated 500-1000 $\times$ in mitochondria. ${ }^{25-27}$

Mice were administered MitoQ $(200 \mu \mathrm{M})$ in their drinking water beginning at 4 weeks of age and continued for 11 weeks. No adverse effects of MitoQ (eg, weight, development) were observed in mice at this dose, consistent with other studies. ${ }^{27-30}$ mROS production in neutrophils was initially examined by MitoSox fluorescence plate
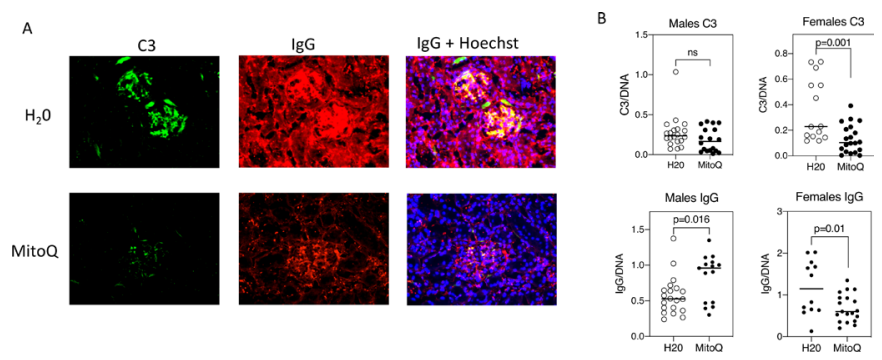

Figure 7 MitoQ reduces kidney immune complexes in female mice. Kidneys were stained for C3 (green), IgG (red) and nuclei by Hoechst stain (blue) and imaged by fluorescence microscopy. The glomerular-associated fluorescence was analysed by pixel quantification in each fluorescence channel using Image J. An example is shown in (A) and summaries all mice analysed is shown in (B). assay. This revealed a reduction in mROS production in MitoQ-treated mice, both spontaneously and following mROS stimulation with the calcium ionophore, A23187 (figure 3A). These findings were observed for both male and female MRL-lpr mice. Consistent with these findings, NET formation was also reduced with MitoQ treatment. In male mice, this was statistically significant for spontaneous NET formation, and in females this was the case following A23187 stimulation (figure 3B).

Further analysis of metabolism in the neutrophils revealed reduced oxygen consumption with MitoQ, both at basal levels and following stimulation of respiration with the mitochondrial uncoupler FCCP (respiratory capacity) (figure 4A). In contrast, glycolysis was largely unchanged, except in the case of female mice where the glycolytic capacity, following addition of ATP synthetase inhibitor oligomycin, was decreased with MitoQ (figure 4B). These results indicate that oral MitoQ modulates lupus neutrophil immunometabolism and reduces their ability to form NETs.

\section{MitoQ in vivo reduces MAVS oligomerisation and serum IFN-I}

CD4 ${ }^{-} \mathrm{CD} 8^{-}$TCR- $\alpha \beta^{+} \mathrm{T}$ cells purified from MitoQ-treated mice revealed reduced levels of MAVS oligomerisation compared with mice receiving water only (figure 5A). This was paralleled by a reduction in serum IFN $\alpha$ (figure $5 \mathrm{~B}$ ). In contrast to the reduction of NET formation and MAVS oligomerisation, MitoQ treatment did not affect lymph node total cell numbers, the distribution of T-cell subsets, including $\mathrm{CD}^{-} \mathrm{CD} 8^{-} \mathrm{TCR}-\alpha \beta^{+} \mathrm{T}$ cells, or $\mathrm{B}$ cells (not shown), nor the titres of serum autoantibodies (figure 6) in male or female MRL-lpr mice. This was somewhat anticipated as MitoQ was not expected to alter events believed to be upstream of mROS production.

\section{Reduced renal injury with MitoQ}

MRL-lpr mice develop immune complex-mediated kidney damage with age. ${ }^{31}{ }^{32}$ Mito $Q$ treatment of female mice caused a reduction of the glomerular deposition of both C3 and IgG (figure 7). This was less apparent in male mice, but male control mice had less C3 and IgG deposition than female control mice, so there was a narrower window in male mice in which to observe a difference with MitoQ. These findings were paralleled by increased urine excretion of creatinine, although without a change in urine albumin (figure 8A) and was also accompanied by a reduction in serum BUN and creatinine in female mice (figure $8 \mathrm{~B}$ ).

\section{DISCUSSION}

The current findings suggest that inhibiting mROS and oxidative stress can mitigate certain aspects of autoimmune disease and organ damage in lupus-prone MRL-lpr mice. It has been appreciated for some time that $\mathrm{T}$ cells and neutrophils in patients with SLE manifest increased levels of ROS, ${ }^{56}$ but the consequences of this on other molecular abnormalities, immune dysregulation or disease manifestations remain largely unexplored. The 
A
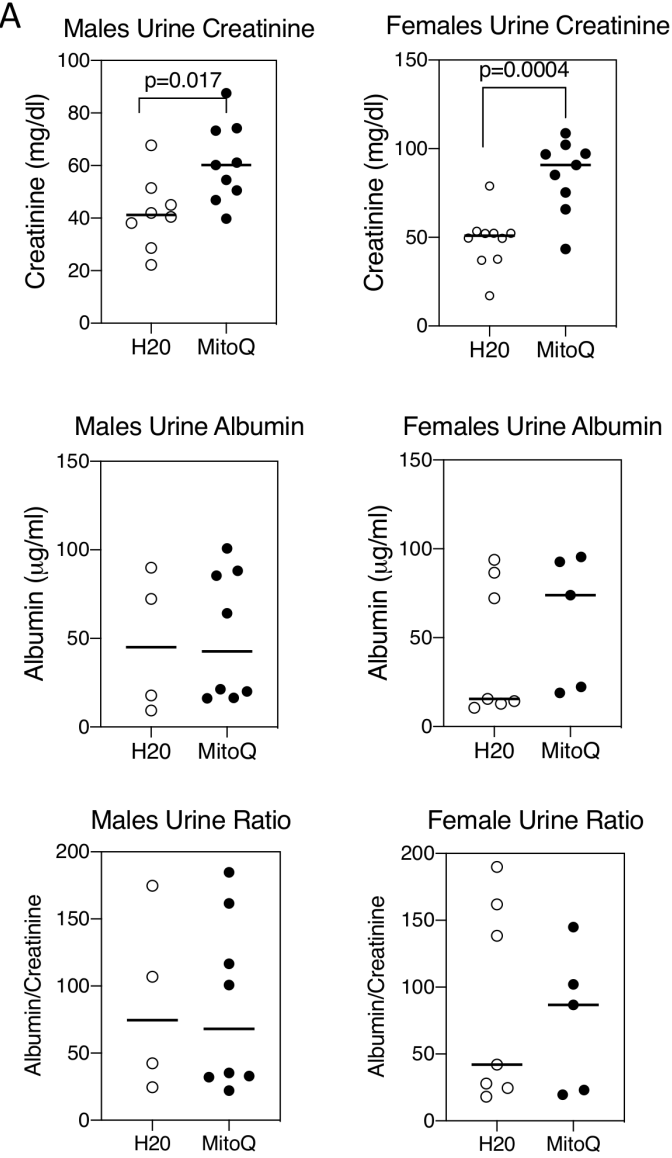
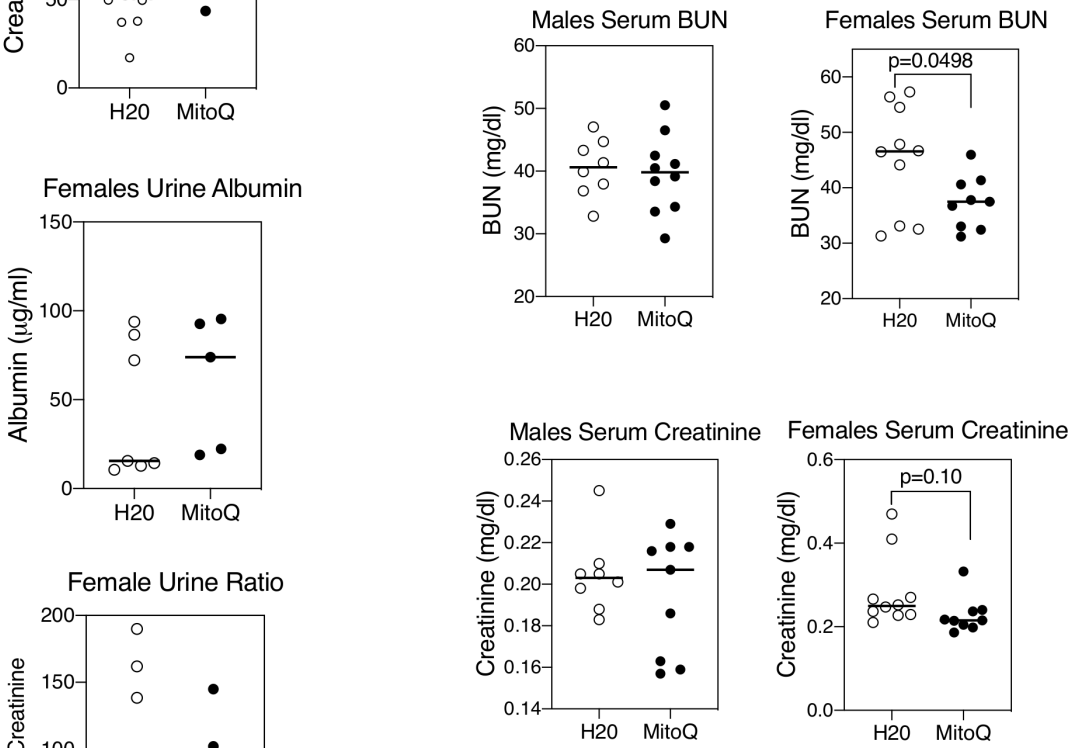

Figure 8 MitoQ improves renal function in female mice. Urine and serum were collected from mice at the end of 11 weeks on water alone or water containing MitoQ. (A) Urine creatinine and albumin concentrations were measured as detailed in the Material and methods section. (B) Serum blood urea nitrogen (BUN) and creatinine concentrations were determined as in the Materials and methods section. Results show mean and individual values for 8-10 mice in each treatment group and of each sex. Statistical analysis was unpaired t-test. Results are representative of two experiments.

current findings reveal that the mitochondria-targeted antioxidant MitoQ can reduce oligomerisation of MAVS and serum IFN-I, mitigate glomerular immune complex formation and help preserve renal function.

The IFN-I gene signature in PBMC is one of the hallmarks of SLE, ${ }^{89}$ yet the mechanism is unclear. The IFN-I signature may be driven in part through engulfment of dead cells by macrophages and dendritic cells. ${ }^{33}$ The increased rate of cell death of neutrophils in patients with SLE, as well as the increased cell death of CD4 ${ }^{-}$ CD8 ${ }^{-}$TCR- $\alpha \beta^{+}$T cells in MRL-lpr mice and human SLE $\mathrm{T}$ cells, may both contribute to the source of dead cells. The released nucleic acids can trigger both ROS and IFN-I production. This is clear from genetic deficiencies in DNA clearance (eg, three prime repair exonuclease (TREX)) which lead to interferonopathies. ${ }^{3435}$

Another source of ROS in human SLE comes from T cells, some of which manifest enlarged mitochondria. ${ }^{5} 14$ We have shown previously that ROS can induce MAVS oligomerisation and IFN-I production in different cell types. ${ }^{15}$ We now observe that, similar to human SLE, mitochondrial enlargement and spontaneous MAVS oligomerisation arises in the MRL-lpr CD $4^{-} \mathrm{CD} 8^{-}$TCR- $\alpha \beta^{+}$subset. This has been attributed to an imbalance of mitochondrial biogenesis ${ }^{36}$ and turnover by mitophagy. ${ }^{37}$ Specifically, our previous studies of SLE T cells have demonstrated human retrovirus endogenous sequence (HRES)-1/rasrelated protein 4 (RAB4)-mediated depletion of dynamin related protein 1 (Drp1), a mediator of mitochondrial fission. ${ }^{36}$ The accumulation of mitochondria in lupus $\mathrm{T}$ cells is sensitive to mTOR blockade, with clinical benefit in patients ${ }^{38}$ and mice $^{39}$ with SLE.

The $\mathrm{CD} 4^{-} \mathrm{CD} 8^{-}$TCR- $\alpha \beta^{+}$subset, which also occurs in human SLE, including inflamed kidneys, ${ }^{40}$ arises from recurrent homeostatic proliferation of $\mathrm{CD}^{+}$precursor T cells. ${ }^{17}{ }^{41} \mathrm{CD}^{-} \mathrm{CD} 8^{-}$TCR- $\alpha \beta^{+} \mathrm{T}$ cells also derive from antigen-specific $\mathrm{CD}^{+} \mathrm{T}$ cells in wild-type mice only when the antigen is presented as a self-antigen. ${ }^{42}$ Gene expression profiling of these wild-type derived $\mathrm{CD} 4^{-} \mathrm{CD} 8^{-}$ TCR- $\alpha \beta^{+}$T cells has revealed upregulation of genes for programmed cell death 1 (PD-1), interleukin 17, IFN $\gamma$, C-X-C Motif Chemokine Ligand 2 (CXCL2) and downregulation of $\mathrm{CD} 127,{ }^{43}$ exactly the same pattern observed in $l p r \mathrm{CD} 4^{-} \mathrm{CD} 8^{-} \mathrm{TCR}-\alpha \beta^{+} \mathrm{T}$ cells. ${ }^{22}$ Thus, these unusual $\mathrm{T}$ cells are not unique to Fas-deficient $l p r$ mice. These findings, in addition to our previous observations that T-cell homeostatic proliferation also leads to the upregulation of genes involved with cytolysis and inflammation ${ }^{22}$ 
adds further importance why it is critical to regulate the homeostatic expansion of $\mathrm{T}$ cells with Fas. In addition to these gene expression changes, our findings also suggest that part of the programme of dysregulated T-cell homeostatic proliferation in $l p r$ mice includes mitochondrial enlargement leading to mROS production and MAVS oligomerisation.

MitoQ has shown therapeutic potential in a variety of conditions in which mROS has been implicated. These include animal models of Alzheimer's disease, ${ }^{29}$ liver fibrosis, ${ }^{30}$ NLR Family Pyrin Domain Containing 3 (NLRP3) inflammasome-mediated colitis, ${ }^{28}$ cardiac ischaemia-reperfusion ${ }^{44}$ and the metabolic syndrome in Apolipoprotein E (ApoE $)^{-/-}$mice..$^{27}$ In human trials, MitoQ prevented inflammatory damage in a phase II study in hepatitis $\mathrm{C}^{45}$ showed no benefit in Parkinson's disease but was well tolerated ${ }^{46}$ and improved vascular function in older adults. ${ }^{47}$ Oxidative stress could be functioning at several levels in SLE, including induction of MAVS oligomerisation ${ }^{15}$ and oxidised mitochondrial DNA release during NET formation, ${ }^{10}$ each contributing to the activation of the IFN-I pathway. In addition, immune complex and complement deposition in renal glomeruli induces oxidative stress in renal epithelium. ${ }^{10}$ Thus, MitoQ may function at several points in reducing oxidative damage in the pathogenesis of SLE.

From our current understanding that Mito $Q$ reduces mROS and oxidative stress and hence events downstream of mROS generation, we would not have anticipated MitoQ to affect events upstream of mROS production. These might include the development of lymphadenopathy of MRL-lpr mice, the generation of $\mathrm{CD} 4^{-} \mathrm{CD} 8^{-}$ TCR- $\alpha \beta^{+}$T cells and the production of autoantibodies, which may result from the absence of cell death occurring during homeostatic proliferation of lymphocytes. ${ }^{1748}$ Consequently, the upregulation of genes during T-cell homeostatic proliferation involved with cytolysis and inflammation, such as granzyme $\mathrm{B}$, perforin and Fasligand, ${ }^{22}$ is likely to still contribute to inflammation during MitoQ therapy. Thus, fully effective therapy for SLE may require a combination of traditional immunosuppression with non-immune antioxidant therapy.

\footnotetext{
Author affiliations

${ }^{1}$ Vermont Center for Immunology and Infectious Diseases, Department of Medicine, University of Vermont Larner College of Medicine, Burlington, VT, USA

${ }^{2}$ Systemic Autoimmunity Branch, National Institute of Arthritis and

Musculoskeletal and Skin Diseases, National Institutes of Health (NIH), Bethesda, MD, USA

${ }^{3}$ Department of Microbiology and Immunology, Upstate Medical University, Syracuse, NY, New York

${ }^{4}$ Rheumatology Clinic, Upstate University Hospital, Syracuse, NY, New York ${ }^{5}$ Department of Pathology and Laboratory Medicine, University of Vermont Larner College of Medicine, Burlington, VT, USA

${ }^{6}$ Renal Diagnostics and Therapeutic Unit, Kidney Diseases Branch, National Institutes of Diabetes and Digestive and Kidney Diseases, Bethesda, Maryland, United States

${ }^{7}$ MRC Mitochondrial Biology Unit, Biomedical Campus, University of Cambridge, Cambridge, CB2 OXY, UK

${ }^{8}$ Department of Medicine, University of Cambridge, Cambridge, CB2 0QQ, UK
}

Acknowledgements We thank Dr Roxana del Rio-Guerra for technical assistance with flow cytometry, as well as the Harry Hood Bassett for flow cytometry and cell sorting facility at the University of Vermont Larner College of Medicine for the use of BD LSR II.

Contributors KAF performed all animal breeding, administration of MitoQ, organ harvest, purification of lymphocyte subsets and flow cytometry, serum creatinine and BUN, and urine albumin/creatinine. LPB performed the ROS and NET formation assays, and immune complex staining of kidneys. IB performed the assays for MAVS oligomerisation. NK and AP performed the autoantibody ELISAs. PCG and DLC performed histological analyses. PSTY performed the serum creatinine analysis. MPM provided the MitoQ and advised on mitochondrial studies. MPM and RCB directed the work.

Funding This work was supported by National Institutes of Health grants Al119979 and GM118228 (to RCB), Al048079, Al072648 and Al122176 (to AP), the Central New York Community Foundation (to AP), the Medical Research Council UK (MC_U105663142) and by a Wellcome Trust Investigator award (110159/Z/15/Z) (to MPM), and the Intramural Research Program at NIAMS (ZIAAR041199). MitoQ was kindly provided by MitoQ, Auckland, New Zealand.

Competing interests MPM helped develop MitoQ and has a commercial interest in MitoQ.

Patient and public involvement Patients and/or the public were not involved in the design, conduct, reporting or dissemination plans of this research.

Patient consent for publication Not required.

Provenance and peer review Not commissioned; externally peer reviewed. Data availability statement All data relevant to the study are included in the article or uploaded as supplementary information. For inquiries, please contact the corresponding author RCB at ralph.budd@med.uvm.edu.

Open access This is an open access article distributed in accordance with the Creative Commons Attribution 4.0 Unported (CC BY 4.0) license, which permits others to copy, redistribute, remix, transform and build upon this work for any purpose, provided the original work is properly cited, a link to the licence is given, and indication of whether changes were made. See: https://creativecommons.org/ licenses/by/4.0/.

\section{ORCID iDs}

Luz P Blanco http://orcid.org/0000-0002-8468-6518

Michael P Murphy http://orcid.org/0000-0003-1115-9618

Mariana J Kaplan http://orcid.org/0000-0003-2968-0815

Ralph C Budd http://orcid.org/0000-0001-8524-8758

\section{REFERENCES}

1 Zucchi D, Elefante E, Calabresi E, et al. One year in review 2019: systemic lupus erythematosus. Clin Exp Rheumatol 2019;37:715-22.

2 Chen L, Morris DL, Vyse TJ. Genetic advances in systemic lupus erythematosus: an update. Curr Opin Rheumatol 2017;29:423-33.

3 Shivakumar S, Tsokos GC, Datta SK. T cell receptor A/B expressing double negative (CD4-CD8-) and CD4+ T helper cells in humans augment the production of pathogenic anti-DNA autoantibodies associated with lupus nephritis. Journal of Immunology 1989;143:103-13.

4 Tsokos GC, Mitchell JP, Juang Y-T. T cell abnormalities in human and mouse lupus: intrinsic and extrinsic. Curr Opin Rheumatol 2003;15:542-7.

5 Gergely P, Grossman C, Niland B, et al. Mitochondrial hyperpolarization and ATP depletion in patients with systemic lupus erythematosus. Arthritis Rheum 2002;46:175-90.

6 Fernandez D, Perl A. Metabolic control of T cell activation and death in SLE. Autoimmun Rev 2009;8:184-9.

7 Yang S-K, Zhang H-R, Shi S-P, et al. The role of mitochondria in systemic lupus erythematosus: a glimpse of various pathogenetic mechanisms. Curr Med Chem 2018. doi:10.2174/09298673266661 81126165139. [Epub ahead of print: 26 Nov 2018] (published Online First: $2018 / 11 / 28)$

8 Crow MK. Type I interferon in systemic lupus erythematosus. Curr Top Microbiol Immunol 2007;316:359-86.

9 Barrat FJ, Crow MK, Ivashkiv LB. Interferon target-gene expression and epigenomic signatures in health and disease. Nat Immunol 2019;20:1574-83.

10 Lood C, Blanco LP, Purmalek MM, et al. Neutrophil extracellular traps enriched in oxidized mitochondrial DNA are interferogenic and contribute to lupus-like disease. Nat Med 2016;22:146-53. 
11 Kaplan MJ. Neutrophils in the pathogenesis and manifestations of SLE. Nat Rev Rheumatol 2011;7:691-9.

12 Villanueva E, Yalavarthi S, Berthier CC, et al. Netting neutrophils induce endothelial damage, infiltrate tissues, and expose immunostimulatory molecules in systemic lupus erythematosus. $J$ Immunol 2011;187:538-52.

13 Garcia-Romo GS, Caielli S, Vega B, et al. Netting neutrophils are major inducers of type I IFN production in pediatric systemic lupus erythematosus. Sci Trans/ Med 2011;3:73ra20.

14 Gergely P, Niland B, Gonchoroff N, et al. Persistent mitochondrial hyperpolarization, increased reactive oxygen intermediate production, and cytoplasmic alkalinization characterize altered IL-10 signaling in patients with systemic lupus erythematosus. J Immunol 2002;169:1092-101.

15 Buskiewicz IA, Montgomery T, Yasewicz EC, et al. Reactive oxygen species induce virus-independent MAVS oligomerization in systemic lupus erythematosus. Sci Signal 2016;9:115.

16 Pothlichet J, Niewold TB, Vitour D, et al. A loss-of-function variant of the antiviral molecule MAVS is associated with a subset of systemic lupus patients. EMBO Mol Med 2011;3:142-52.

17 Fortner KA, Budd RC. The death receptor Fas (CD95/Apo-1) mediates the deletion of T lymphocytes undergoing homeostatic proliferation. J Immunol 2005;175:4374-82.

18 Alberti S, Halfmann R, King O, et al. A systematic survey identifies prions and illuminates sequence features of prionogenic proteins. Cell 2009;137:146-58.

19 Yuen PST, Dunn SR, Miyaji T, et al. A simplified method for HPLC determination of creatinine in mouse serum. Am J Physiol Renal Physiol 2004;286:F1116-9.

20 Landolfi MM, Van Houten N, Russell JQ, et al. CD2-CD4-CD8lymph node T lymphocytes in MRL Ipr//pr mice are derived from a CD2+CD4+CD8+ thymic precursor. J Immunol 1993;151:1086-96.

21 Mixter PF, Russell JQ, Durie FH, et al. Decreased CD4-CD8- TCR-ab cells in /pr/lpr mice lacking b2-microglobulin. Journal of Immunology 1995; 154:2063-74.

22 Fortner KA, Bond JP, Austin JW, et al. The molecular signature of murine $T$ cell homeostatic proliferation reveals both inflammatory and immune inhibition patterns. J Autoimmun 2017;82:47-61.

23 Secinaro M, Fortner KA, Dienz O, et al. Glycolysis promotes caspase-3 activation in lipid rafts in T cells. Cell Death and Disease 2017.

24 Secinaro MA, Fortner KA, Collins C, et al. Glycolysis induces MCJ expression that links $T$ cell proliferation with caspase-3 activity and death. Front Cell Dev Biol 2019;7:28.

25 Murphy MP. Understanding and preventing mitochondrial oxidative damage. Biochem Soc Trans 2016;44:1219-26.

26 McLachlan J, Beattie E, Murphy MP, et al. Combined therapeutic benefit of mitochondria-targeted antioxidant, MitoQ10, and angiotensin receptor blocker, losartan, on cardiovascular function. $J$ Hypertens 2014;32:555-64.

27 Mercer JR, Yu E, Figg N, et al. The mitochondria-targeted antioxidant MitoQ decreases features of the metabolic syndrome in ATM+/-/ ApoE-/- mice. Free Radic Biol Med 2012;52:841-9.

28 Dashdorj A, Jyothi KR, Lim S, et al. Mitochondria-Targeted antioxidant MitoQ ameliorates experimental mouse colitis by suppressing NLRP3 inflammasome-mediated inflammatory cytokines. BMC Med 2013;11:178.

29 McManus MJ, Murphy MP, Franklin JL. The mitochondria-targeted antioxidant MitoQ prevents loss of spatial memory retention and early neuropathology in a transgenic mouse model of Alzheimer's disease. J Neurosci 2011;31:15703-15.
30 Rehman H, Liu Q, Krishnasamy Y, et al. The mitochondria-targeted antioxidant Mito Q attenuates liver fibrosis in mice. Int J Physiol Pathophysiol Pharmacol 2016;8:14-27.

31 Cohen PL, Eisenberg RA. Lpr and gld: single gene models of systemic autoimmunity and lymphoproliferative disease. Annu Rev Immunol 1991;9:243-69.

32 Nickerson KM, Cullen JL, Kashgarian M, et al. Exacerbated autoimmunity in the absence of TLR9 in MRL.Fas(lpr) mice depends on Ifnar1. J Immunol 2013;190:3889-94.

33 Lande R, Ganguly D, Facchinetti V, et al. Neutrophils activate plasmacytoid dendritic cells by releasing self-DNA-peptide complexes in systemic lupus erythematosus. Sci Transl Med 2011;3:73ra19.

34 Crow YJ, Hayward BE, Parmar R, et al. Mutations in the gene encoding the $3^{\prime}-5^{\prime}$ DNA exonuclease TREX1 cause Aicardi-Goutières syndrome at the AGS1 locus. Nat Genet 2006;38:917-20.

35 Lee-Kirsch MA, Chowdhury D, Harvey S, et al. A mutation in TREX1 that impairs susceptibility to granzyme A-mediated cell death underlies familial chilblain lupus. J Mol Med 2007;85:531-7.

36 Caza TN, Fernandez DR, Talaber G, et al. HRES-1/RAB4-mediated depletion of Drp1 impairs mitochondrial homeostasis and represents a target for treatment in SLE. Ann Rheum Dis 2014;73:1888-97.

37 Nagy G, Barcza M, Gonchoroff N, et al. Nitric oxide-dependent mitochondrial biogenesis generates $\mathrm{Ca} 2+$ signaling profile of lupus $\mathrm{T}$ cells. J Immunol 2004;173:3676-83.

38 Lai Z-W, Kelly R, Winans T, et al. Sirolimus in patients with clinically active systemic lupus erythematosus resistant to, or intolerant of, conventional medications: a single-arm, open-label, phase 1/2 trial. Lancet 2018;391:1186-96.

39 Oaks Z, Winans T, Caza T, et al. Mitochondrial dysfunction in the liver and antiphospholipid antibody production precede disease onset and respond to rapamycin in lupus-prone mice. Arthritis Rheumatol 2016;68:2728-39.

40 Crispín JC, Oukka M, Bayliss G, et al. Expanded double negative T cells in patients with systemic lupus erythematosus produce IL-17 and infiltrate the kidneys. J Immunol 2008;181:8761-6.

41 Crispín JC, Tsokos GC. Human TCR-alpha beta+ CD4- CD8- T cells can derive from CD8+ T cells and display an inflammatory effector phenotype. J Immunol 2009;183:4675-81.

42 Rodríguez-Rodríguez N, Apostolidis SA, Penaloza-MacMaster P, et al. Programmed cell death 1 and Helios distinguish TCR- $\alpha \beta+$ double-negative (CD4-CD8-) T cells that derive from self-reactive CD8 T cells. J Immunol 2015;194:4207-14.

43 Rodríguez-Rodríguez N, Apostolidis SA, Fitzgerald L, et al. Proinflammatory self-reactive T cells are found within murine TCR- $\alpha \beta(+)$ CD4(-) CD8(-) PD-1(+) cells. Eur J Immunol 2016;46:1383-91.

44 Adlam VJ, Harrison JC, Porteous CM, et al. Targeting an antioxidant to mitochondria decreases cardiac ischemia-reperfusion injury. Faseb J 2005;19:1088-95.

45 Gane EJ, Weilert F, Orr DW, et al. The mitochondria-targeted antioxidant mitoquinone decreases liver damage in a phase II study of hepatitis C patients. Liver Int 2010;30:1019-26.

46 Snow BJ, Rolfe FL, Lockhart MM, et al. A double-blind, placebocontrolled study to assess the mitochondria-targeted antioxidant MitoQ as a disease-modifying therapy in Parkinson's disease. Mov Disord 2010;25:1670-4.

47 Rossman MJ, Santos-Parker JR, Steward CAC, et al. Chronic supplementation with a mitochondrial antioxidant (MitoQ) improves vascular function in healthy older adults. Hypertension 2018;71:1056-63.

48 Shlomchik M, Mascelli M, Shan $\mathrm{H}$, et al. Anti-Dna antibodies from autoimmune mice arise by clonal expansion and somatic mutation. $J$ Exp Med 1990;171:265-92. 


\section{Correction: Targeting mitochondrial oxidative stress with Mito $Q$ reduces NET formation and kidney disease in lupus-prone MRL-lpr mice}

Fortner KA, Blanco LP, Buskiewicz I, et al. Targeting mitochondrial oxidative stress with MitoQ reduces NET formation and kidney disease in lupus-prone MRL-lpr mice. Lupus Sci Med 2020;7:e00387.

The published version misspelled co-author's name as Andreas Perl. The correct name should be Andras Perl.

\section{() \\ OPEN ACCESS}

Open access This is an open access article distributed in accordance with the Creative Commons Attribution 4.0 Unported (CC BY 4.0) license, which permits others to copy, redistribute, remix, transform and build upon this work for any purpose, provided the original work is properly cited, a link to the licence is given, and indication of whether changes were made. See: https://creativecommons.org/licenses/by/4.0/.

Lupus Sci Med 2020;7:e000387corr1. doi:10.1136/lupus-2020-000387corr1

A) Check for updates 